\title{
Cutaneous Plasmacytoma Associated with Squamous Cell Carcinoma of the Sacro-Coccygeal Region
}

\author{
Gianfranco Orlandino ${ }^{1}$, Roberto Guariglia ${ }^{2}$, Juri Tassinari ${ }^{1}$, Luigina Di \\ Giovannantonio $^{3}$, Giulia Vita ${ }^{3}$, Pellegrino Musto ${ }^{2}$ and Tommaso Fabrizio ${ }^{1}$ \\ ${ }^{1}$ Unit of Plastic and Reconstructive Surgery, IRCCS - Centro di Riferimento Oncologico Basilicata. \\ Rionero in Vulture (PZ), Italy \\ ${ }^{2}$ Unit of Haematology and Stem Cell Transplantation, IRCCS - Centro di Riferimento Oncologico \\ Basilicata. Rionero in Vulture (PZ), Italy \\ ${ }^{3}$ Unit of Histopathology. IRCCS - Centro di Riferimento Oncologico Basilicata. Rionero in Vulture \\ (PZ), Italy
}

Correspondence should be addressed to: Gianfranco Orlandino; gianfranco.orlandino@crob.it

Received 14 November 2012; Accepted 19 December 2012; Published 29 May 2013

Academic Editor: Martin Busch

Copyright (c) 2013 Gianfranco Orlandino, Roberto Guariglia, Juri Tassinari, Luigina Di Giovannantonio, Giulia Vita, Pellegrino Musto and Tommaso Fabrizio. Distributed under Creative Commons CC-BY 3.0

\begin{abstract}
We describe the case of a patient who received the diagnosis of squamous cell carcinoma of the radio-treated skin of the sacrococcygeal region where an extramedullary plasmacytoma had been identified one year before. We think that the plasmacytoma was born by a malignant transformation of a host-inflammatory reaction to a not-detected epithelial tumor. It can also hypothesized that plasma-cell dyscrasia or post-radiation infiammatory reaction had promoved the squamous cell carcinoma occurrence.
\end{abstract}

Keywords: Extramedullary plasmacytoma, squamous cell carcinoma, radiotherapy, skin island flaps.

\section{Introduction}

Extramedullary plasmacytoma (EMP) is a plasma-cell tumour that origins from soft tissues. Diagnosis requires a biopsy-proven monoclonal plasma cell infiltration of a single site without evidence of systemic disease (bone marrow involvement, hypercalcemia, lytic bone lesions, anemia or renal disease); a monoclonal paraprotein is detected in the serum and/or urine in fewer than $25 \%$ of patients [Dimopoulos, 2002; UKMF, 2004; Weber, 2005]. Since EMP, as well as solitary bone plasmacytoma, is a highly radiosensitive neoplasm, the treatment of choice is radiotherapy given at the dose of 35-45 Gy. Chemotherapy and surgical procedures are

Cite this Article as: Gianfranco Orlandino, Roberto Guariglia, Juri Tassinari, Luigina Di Giovannantonio, Giulia Vita, Pellegrino Musto and Tommaso Fabrizio (2013), "Cutaneous Plasmacytoma Associated with Squamous Cell Carcinoma of the Sacro-Coccygeal Region," International Journal of Case Reports in Medicine, Vol. 2013 (2013), Article ID 913279, DOI: 10.5171/2013.913279 
rarely necessary because no clear evidence exists that they can reduce the probability of progression to multiple myeloma or increase disease-free survival [Weber, 2005; Chao, 2005].

Approximately $90 \%$ of EMP occur in the head and neck mucosa, especially in the upper respiratory tract including nasal cavity, sinuses and oropharynx, while skin represents an infrequent localization [UKMF, 2004].

What we present here is an unusual case of cutaneous EMP associated with squamous cell carcinoma (SCC) localized at sacrococcygeal region.

\section{Case Report}

A 56-year-old male was admitted at the Onco-Hematology Department of our
Institution on September, 2006, because of a large $(10 \mathrm{~cm})$, infiltrating skin lesion of the sacral region. An incisional biopsy was performed and the histo-pathological examination releaved the presence of a diffuse dermic-hypodermic plasma cell neoplastic infiltration [Figure 1], with lambda light-chain restriction. The patient was further investigated: the absence of bone marrow plasma cell infiltration, lytic bone lesions at skeletal survey, hypercalcemia, anemia and renal disease supported the diagnosis of solitary, cutaneous EMP. No M-component was detected at serum and urine immunofixation. On December, 2006, the patient started a radiotherapy treatment with a dose of 40 Gy subdivided in 20 fractions of $200 \mathrm{cGy}$ each.

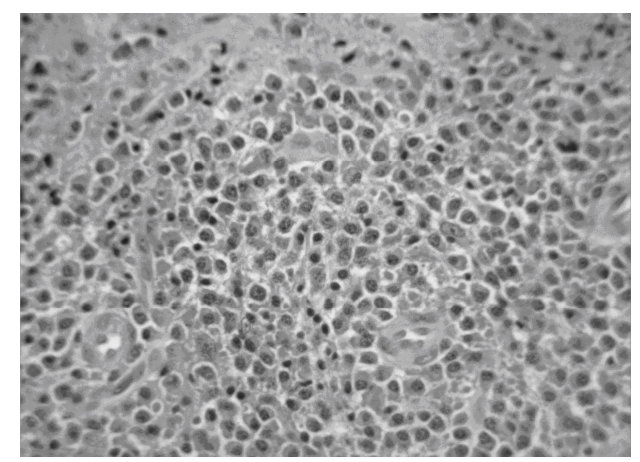

\section{Fig. 1: Extramedullary Plasmacytoma of the Sacro-Coccygeal Region; Ematossilin- Eosin} Coloration (A) and Immunohistochemistry for Lambda Chain (B), 40X Magnification

A few weeks after the end of radiotherapy, a suppurative inflammatory infiltration developed at the cicatrix level. The patient was initially treated with systemic antibiotic therapy and local medications; however, because of the inefficacy of this therapy, he went through a surgical drainage of the abscess. The removed material was addressed to pathology examination, which excluded the relapse of the plasma cell disease or the presence of other neoplastic lesions, exclusively confirming an inflammatory process.

Three months later, however, the skin lesion had significantly worsened: it was 20 x $25 \mathrm{~cm}$ large, hard, fixed on the lower levels, with pus that still discharged from a fistulous tract. Therefore, the patient was addressed again to the operating theatre. The lesion and all the involved soft tissues were completely removed. The histological examination revealed the presence of an invasive, differentiated SCC with surgical margins in healthy tissue and no signs of EMP [Figure 2]. In order to repair the recipient zone, two very large skin and soft tissues island flaps, pedicled on the perforating vessels of the superior and inferior gluteus arteries, were harvested and tiled on the middle line [Figure 3]. To date, after forty-four months of follow-up, the patient does not show any evidence of relapse of both neoplasms. 


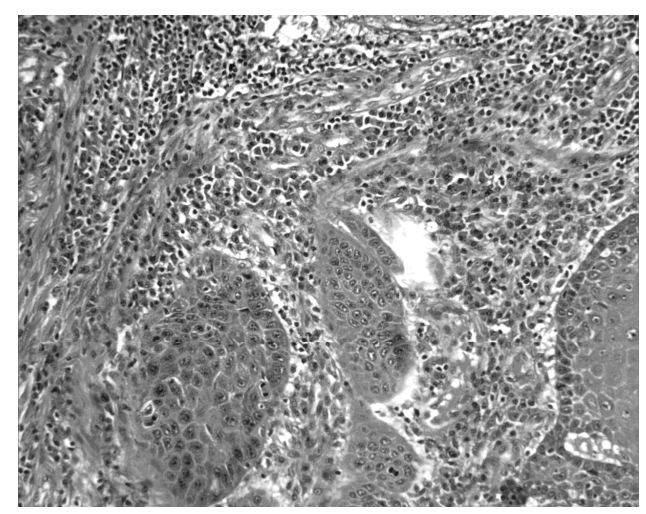

Fig. 2: Squamous Cell Carcinoma of the Sacro-Coccygeal Region; Ematossilin-Eosin Coloration, 20X Magnification

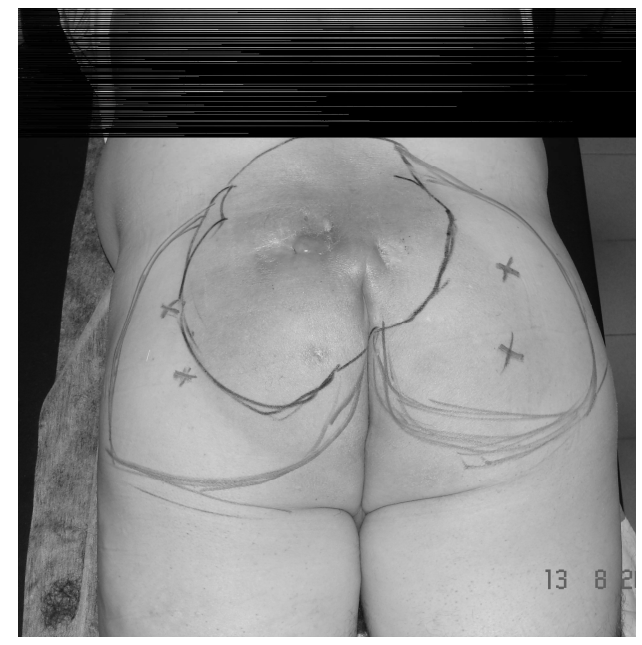

Fig. 3: Planning of Removal and Reconstruction Procedures (A); Recipient Zone with Complete Healing after Plastic Surgery (B)

\section{Discussion}

Simultaneous occurrence of EMP and SCC is rare and has been only occasionally reported at lung, tonsil and breast level [Dasmahapatra, 1986; Junquera, 2009; Cao, 2009]. In addition, Jayagopal et al. described a case of SCC of the skin where EMP was evidenced at the margin of the specimen. These authors supposed that EMP might have reflected a malignant transformation of a host-immune reaction to a previous SCC [Jayagopal, 2004].

In our patient, SCC was identified on samples obtained by a large excision of a skin tract previously treated with radiotherapy for EMP. The occurrence of SCC on irradiated skin has been described as a possible mutagen effect of radiotherapy [Van Vloten, 1987]. In our case, however, the time from treatment to the identification of SCC was probably too short to explain this linkage.

The hypothesis that the two neoplasms were synchronous is also weak, since SCC and EMP are both rare, as single disease, at sacro-coccygeal region; on the other hand, no simultaneous evidence of both disease was shown in our patient. Even if the hypothesis that EMP is due to a malignant transformation of a preavius reactive plasmacellular infiltration is the most acceptable, we favour the alternative possibility that EMP by itself, in combination with post-radiation persistent chronic inflammatory reaction, could have 
played a (cytokine-mediated?) local "oncologic promoter" role, inducing the rapid development of SCC.

\section{References}

Cao, S., Kang, H. G., Liu, Y. X. \& Ren, X. B. (2009). "Synchronous Infiltrating Ductal Carcinoma and Primary Extramedullary Plasmacytoma of the Breast," World J Surg Oncol 2009; 7: 43.

Chao, M. W., Gibbs, P., Wirth, A., et al. (2005). "Radiotherapy in the Management of Solitary Extramedullary Plasmacytoma," Intern Med J 2005; 35: 211-215.

Dasmahapatra, H. K., Candlish, W. \& Davidson, K. G. (1986). "Combined Plasmacytoma and Squamous Cell Carcinoma," J Thorac Cardiovasc Surg 1986; 34: 403-405.

Dimopoulos, M. A. \& Hamilos, G. (2002). "Solitary Bone Plasmocytoma and Extramedullary Plasmocytoma," Curr Treat Options Oncol 2002; 3: 255-259.

Jayagopal, S., Berry, M. G., Ross, G., et al. (2004). "A Case of Squamous Cell Carcinoma Associated with Plasmacytoma," Br J Plast Surg 2004; 57: 172-173.

Junquera, L., Gallego, L., Torre, A., et al. (2009). "Synchronous Oral Squamous Cell Carcinoma and Extramedullary Plasmacytoma af the Tonsil," Oral Surg Oral Pathol Oral Radiol Endod 2009; 108: 413416.

Soutar, R., Lucraft, H., Jackson, G., Reece, A., Bird, J., Low, E. \& Samson, D. Guidelines Working Group of the UK Myeloma Forum (UKMF). (2004). "Guidelines on the Diagnosis and Management of Solitary Plasmacytoma of Bone and Solitary Extramedullary Plasmacytoma," $\mathrm{Br} \mathrm{J}$ Haematol 2004; 124: 717-726.

Van Vloten, W. A., Hermans, J. \& Van Daal, W. A. J. (1987). "Radiation-Induced Skin Cancer and Radiodermatitis of the Head and Neck," Cancer 1987; 59: 411-414.
Weber, D. M. (2005). "Solitary Bone and Extramedullary Plasmacytoma," Hematology - American Society of Hematology. Educational Program Book 2005: 373-376.

Gianfranco Orlandino, Roberto Guariglia, Juri Tassinari, Luigina Di Giovannantonio, Giulia Vita, Pellegrino Musto and Tommaso Fabrizio (2013), International Journal of Case Reports in Medicine,

DOI: $10.5171 / 2013.913279$ 